\title{
ACTIVIDAD ANTIOXIDANTE, POLIFENOLES Y FLAVONOIDES DE Coffea arabica de CINCO REGIONES PERUANAS
}

\author{
Silvia Suárez Cunza ${ }^{a}$, Elvi Alfaro Pillihuaman ${ }^{\mathrm{b}}$, Emilio German Ramírez Roca*b $^{*}$
}

\begin{abstract}
RESUMEN
El café preparado de diversas formas contiene fenoles y flavonoides con actividad antioxidante y con beneficios para la salud. El café peruano cuenta con escasa información sobre sus propiedades antioxidantes. Los objetivos fueron evaluar la capacidad antioxidante total in vitro, y el contenido de fenoles totales y flavonoides de cafés procedentes de los valles de Chanchamayo (Junín), Tutumbaro (Ayacucho), Oventeni (Ucayali), Jaén (Cajamarca) y Quillabamba (Cusco). Las muestras se prepararon por infusión al $2 \%$ p/v. Se determinaron sólidos solubles por refractometría y densidad aparente por gravimetría; fenoles totales con el reactivo de Folin-Ciocalteau y flavonoides según Zhishen. La capacidad antioxidante total se evaluó empleando DPPH, ABTS.+ y FRAP, los resultados se expresan como capacidad antioxidante equivalente a Trolox (TEAC) y como equivalente a ácido ascórbico (AAEAC). Se encontraron 2,8-3,0 mmol EAG/g de fenoles totales, los flavonoides fueron 135-155 $\mu \mathrm{mol}$ $\mathrm{ECq} / \mathrm{g}$. Los resultados de TEAC con DPPH estuvieron entre $44-52 \mathrm{mg} / \mathrm{g}$ y para AAEAC entre 41-50 mg/g; con el ABTS, la TEAC fue de 55-62 mg/g y AAEAC fue entre 47-52 $\mathrm{mg} / \mathrm{g}$; hubo diferencia significativa $(\mathrm{p}<0,05)$ para el DPPH y ABTS entre Junín y Cusco. En el FRAP, estuvieron entre 493 y $540 \mu \mathrm{mol} \mathrm{EqFeSO} 4 / \mathrm{g}$. No hubo diferencias significativas. Se concluye que los extractos acuosos de los granos tostados y molidos de Coffea arabica procedentes de los cinco valles poseen efecto antioxidante in vitro en asociación con el contenido de fenoles totales y flavonoides, la diferencia en capacidad antioxidante no es relevante. Este comportamiento es comparable con resultados reportados en la literatura internacional.
\end{abstract}

Palabras clave: Coffea arabica, café, antioxidantes, polifenoles, flavonoides.

\footnotetext{
a Centro de Investigación de Bioquímica y Nutrición, Facultad de Medicina, UNMSM - Lima. Avenida Grau 755, Lima Cercado, Lima 01.

b Escuela de Farmacia y Bioquímica, Facultad de Ciencias de la Salud, UNSCH - Ayacucho. Av. Independencia s/n. Cuidad Universitaria. Ayacucho.*E-mail: Emilio.ramirez@unsch.edu.pe
} 


\title{
ANTIOXIDANT ACTIVITY, POLYPHENOLS AND FLAVONOIDS OF Coffea arabica FROM FIVE PERUVIAN REGIONS
}

\begin{abstract}
Coffee prepared in various ways contains phenols and flavonoids with antioxidant activity and health benefits. Peruvian coffee has little information on its antioxidant properties. The objectives were to evaluate the total antioxidant capacity in vitro and the content of total phenols and flavonoids; then compare the coffees from the valleys of Chanchamayo (Junín), Tutumbaro (Ayacucho), Oventeni (Ucayali), Jaén (Cajamarca) and Quillabamba (Cusco). Samples were prepared by $2 \% \mathrm{w} / \mathrm{v}$ infusion. Soluble solids were determined by refractometry and apparent density by gravimetry; total phenols with the Folin-Ciocalteau reagent and flavonoids according to Zhishen. The total antioxidant capacity was evaluated using DPPH, ABTS. + and FRAP, the results are expressed as Trolox equivalent antioxidant capacity (TEAC) and as Ascorbic Acid equivalent antioxidant capacity (AAEAC). Total phenol results were 2.8-3.0 mmol GAE/g, flavonoids were $135-155 \mu \mathrm{mol} \mathrm{ChE/g}$. The results of TEAC with DPPH were between $44-52 \mathrm{mg} / \mathrm{g}$ and between $41-50 \mathrm{mg} / \mathrm{g}$ for AAEAC; with ABTS between 55-62 mg TEAC/g and between 47-52 mg AAEAC/g; There was a significant difference $(\mathrm{p}<0.05)$ for the DPPH and ABTS between Junín and Cusco. In the FRAP, between 493 and $540 \mu \mathrm{mol} \mathrm{EqFeSO} 4 / \mathrm{g}$. There were no significant differences. It is concluded that the aqueous extracts of the roasted and ground grains of Coffea arabica from the five valleys have an antioxidant effect in vitro in association with the content of total phenols and flavonoids, the difference in antioxidant capacity is not relevant. This behavior is comparable with results reported in the international literature.
\end{abstract}

Key words: Coffea arabica, coffee, antioxidants, polyphenols, flavonoids.

\section{INTRODUCCIÓN}

El consumo de café parece, en general, seguro dentro de los niveles habituales de consumo. Preparado de diversas formas contiene compuestos bioactivos no enzimáticos con capacidad antioxidante, reconocido además por sus beneficios para la salud ${ }^{1}$. El contenido de los compuestos bioactivos no enzimáticos incluye el alcaloide cafeína, además de ácidos fenólicos, principalmente los ácidos clorogénico, elágico y cafeico; también están presentes flavonoides; variando el contenido de estos componentes entre especies y lugar de origen, proporcionándole al café la calidad de alimento funcional y nutracéutico. Debido a la variedad de compuestos bioactivos no enzimáticos, actualmente se conoce que este producto agroalimentario posee una serie de propiedades como actividad antioxidante, anticarcinogénico y antimutagénica ${ }^{2}$.

La composición, así como sus propiedades fisicoquímicas y organolépticas dependen de diversos factores, los edáficos, la altura, las condiciones climáticas, la radiación solar y otros. 
El territorio nacional tiene tal diversidad de condiciones que favorecen la producción del café de modo tal que el $64 \%$ de las regiones producen este recurso vegetal ${ }^{3}$. El café producido hasta su preparación, como bebida, sufre modificaciones en composición y propiedades con el tratamiento y procesamiento poscosecha, las propiedades antioxidantes, por lo tanto, pueden variar sin que ello signifique necesariamente pérdida, porque eventualmente puede aumentar por la formación de otros compuestos con actividad antioxidante, como los productos de Maillard ${ }^{4}$.

En el Perú, el café se cultiva a altitudes desde los 600 hasta los 1800 metros, el $75 \%$ de las zonas de cultivo se encuentran por encima de los 1000 metros sobre el nivel del mar ${ }^{5}$. El Perú es reconocido como un país cafetalero en términos de calidad; sin embargo, los estudios sobre el café cultivado en el Perú están orientados principalmente a los aspectos de cultivo, de procesamiento y calidad organoléptica ${ }^{6}$; siendo escasos los estudios sobre las propiedades funcionales de sus compuestos bioactivos no enzimáticos. El estudio de las propiedades antioxidantes in vitro, de diversas variedades de café que se cultivan en el territorio nacional, es importante para su valoración, más allá de las propiedades fisicoquímicas y sensoriales.

La importancia de estas propiedades fueron la base de los objetivos del presente estudio, la evaluación de las propiedades antioxidantes y la comparación de estas propiedades de los granos de café tostados provenientes de cinco regiones del país, con el fin de hallar un denominador común en su comportamiento, que pueda contribuir a una mejor valoración en su biocomercio. Adicionalmente, la información generada permite una comparación con otros estudios en granos de café a nivel internacional.

\section{PARTE EXPERIMENTAL}

\section{Obtención de las muestras}

Se obtuvo un kilogramo de semilla con cáscara de café orgánico de la especie Coffea arabica provenientes de los valles Tutumbaro (Ayacucho, temperatura media anual $24,4^{\circ} \mathrm{C}$, altitud $1760 \mathrm{~m}$ ), Chanchamayo (Junín, temperatura media anual $24,6^{\circ} \mathrm{C}$, altitud $700 \mathrm{~m}$ ), Oventeni (Ucayali, temperatura media anual $26,1^{\circ} \mathrm{C}$, altitud $1336 \mathrm{~m}$ ), Jaén (Cajamarca, temperatura media anual $24,5^{\circ} \mathrm{C}$, altitud $729 \mathrm{~m}$ ) y Quillabamba (Cusco, temperatura media anual $23,6^{\circ} \mathrm{C}$, altitud $1047 \mathrm{~m}$ ) del Perú, en temporada de cosecha en el mes de marzo de 2018. En todos los casos se realizó un muestreo por conveniencia.

\section{Diseño metodológico}

El tipo de estudio fue cuantitativo mediante un diseño observacional analítico que incluye comparaciones de los granos de diversa procedencia y asociaciones de los parámetros evaluados. El análisis de los resultados se realizó aplicando ANOVA y como prueba de pares poshoc, Tukey, se consideró significativo para un valor $\mathrm{p}<0,05$. La asociación se analizó mediante la correlación de Pearson. Se empleó el programa EXCEL 2016. 


\section{Preparación de las muestras y los extractos acuosos}

Las semillas fueron lavadas secuencialmente con agua destilada, luego con una solución de hipoclorito de sodio al $0,1 \%$, se dejó por 5 minutos y finalmente con agua bidestilada. Se dejó secar a temperatura ambiente por una hora; a continuación, se realizó la separación de la cáscara. Los granos pelados fueron sometidos a tostación en una máquina tostadora de café de acero inoxidable a una temperatura de $200-220^{\circ} \mathrm{C}$ durante $8-12$ minutos, posteriormente se procedió a reducir su tamaño en un molino de café, igualmente de acero inoxidable. Los granos tostados y molidos fueron desecados en la estufa a $37^{\circ} \mathrm{C}$ durante 24 horas, se uniformizó la humedad en un rango de $11-12,5 \%$. El producto de este proceso se usó para la preparación de los extractos acuosos. Se prepararon extractos al 2,0 \% con agua bidestilada a $80^{\circ} \mathrm{C}$ y agitación por 30 minutos, se procedió a filtrar. El filtrado se usó para los análisis de antioxidantes y cuantificación de fenoles totales y flavonoides.

\section{Determinación de propiedades físicas}

Se evalúo densidad aparente, masa seca y sólidos solubles. La densidad aparente se determinó por gravimetría, se midió $1 \mathrm{~mL}$ del extracto y se determinó el peso en una balanza analítica de sensibilidad $0,0001 \mathrm{~g}$. Se llevó a sequedad en una estufa hasta peso constante y la diferencia de pesos correspondió a la masa seca. Los sólidos solubles se midieron en un refractómetro digital, de sensibilidad 0,01 brix con termómetro incluido. Todos los análisis se realizaron por triplicado de muestras independientes a una temperatura de $25^{\circ} \mathrm{C}$.

\section{Determinación de compuestos bioactivos no enzimáticos: polifenoles y flavonoides}

Contenido de polifenoles. Fueron cuantificados con el reactivo Folin Ciocalteu? ${ }^{7}$ El tubo de reacción de un mililitro contenía $0,1 \mathrm{ml}$ de muestra o estándar, se le añadió $0,5 \mathrm{~mL}$ del reactivo Folin Ciocalteu diluido 1:10 y una solución de Na2CO3 al 7,5 \%. Después de 30 minutos se leyó a $765 \mathrm{~nm}$. Se preparó tubo blanco de reactivos. El estándar fue ácido gálico de $20 \mu \mathrm{g} / \mathrm{mL}$. Se realizaron tres repeticiones y los resultados se expresan en mmol EAG/g.

Contenido de flavonoides (CF). La cuantificación de los flavonoides se realizó con la técnica de Zhishen et al $^{8}$. Basado en la propiedad complejante de los flavonoides frente el aluminio $(2,5 \%)$ en presencia de $\mathrm{NaNO}_{2}(5 \%)$ en medio alcalino (NaOH 1 M). La absorbancia se leyó a $510 \mathrm{~nm}$. La curva de catequina se preparó de 50-250 $\mu \mathrm{M}$. El tubo de reacción contenía 0,5 $\mathrm{mL}$ de la muestra de extracto o el patrón de catequina, $150 \mu \mathrm{L}$ de nitrito de sodio, después de 5 minutos, se agregó $250 \mu \mathrm{L}$ de cloruro de aluminio, reposo por 6 minutos y finalmente 250 $\mu \mathrm{L}$ de $\mathrm{NaOH}$, después de 10 minutos se leyeron. Se preparó un blanco de reactivo y un blanco de muestra. Se realizaron tres repeticiones y los resultados se expresan como equivalentes de catequina $(\mu \mathrm{mol} \mathrm{ECq} / \mathrm{g})$.

\section{Medición de la Capacidad Antioxidante Total (CAT)}

La CAT se midió con el radical ABTS.+ con la técnica de Re et al ${ }^{9}$. El radical se preparó a partir del compuesto ABTS [ácido 7-azinobis- (ácido 3-etilbenzotiazolina-6-sulfónico)] 7 $\mathrm{mM}$ en reacción con persulfato de potasio $2,45 \mathrm{mM}$, durante $16 \mathrm{~h}$ alejado de la luz. La presencia del antioxidante reduce la absorbancia inicial de 0,7+0,02 medido a $734 \mathrm{~nm}$. Se preparó tubos de control, blanco y muestra; los tubos tenían un volumen final de 1,0 
$\mathrm{mL}$ y la muestra de extracto fue de $20 \mu \mathrm{L}$. Se realizaron tres repeticiones y las lecturas se realizaron después de 7 minutos de reacción en un ambiente alejado de la luz. Los resultados se expresan como capacidad antioxidante equivalente al Trolox (TEAC-ABTS) y capacidad antioxidante equivalente al ácido ascórbico (AAEAC-ABTS). La curva de calibración de Trolox fue de 0,5 a 2,5 $\mu \mathrm{g} / \mathrm{mL}$ y la del ácido ascórbico de 1,0 a 4,0 $\mu \mathrm{g} / \mathrm{mL}$.

CAT con el radical libre DPPH. El radical libre difenil picril hidrazilo (DPPH) de color púrpura fue preparado en etanol grado analítico para una lectura inicial de 0,6 + 0,02 medido a $517 \mathrm{~nm}$ (Joyeux et al) ${ }^{10}$. Se prepararon tubos de control, blanco y muestra; los tubos tenían un volumen final de $1,2 \mathrm{~mL}$ y la muestra de extracto fue de $0,4 \mathrm{~mL}$, se dejaron reaccionar alejados de la luz y después de 30 minutos se midió la absorbancia. Los resultados se expresan como capacidad antioxidante equivalente al Trolox (TEAC-DPPH) y capacidad antioxidante equivalente al ácido ascórbico (AAEAC-DPPH). La curva de calibración de Trolox fue de 0,5 a 2,5 $\mu \mathrm{g} / \mathrm{mL}$ y la del ácido ascórbico de 1,0 a $4,0 \mu \mathrm{g} / \mathrm{mL}$.

CAT medido como el potencial antioxidante de reducción de $F e^{+3}$ (FRAP). Se evaluó según la metodología de Benzie et $a l^{11}$. La molécula 2,4,6-tripiridil-s-triazina (TPTZ) actúa como un ligando altamente estabilizador de $\mathrm{Fe}^{+2}$ ante la presencia de un antioxidante que reduce el ion $\mathrm{Fe}^{+3}$, en tampón acetato $300 \mathrm{mM}$, pH 3,6. La reacción se midió por el aumento de la absorbancia a la longitud de onda de $593 \mathrm{~nm}$. El tubo contenía $50 \mu \mathrm{L}$ del extracto acuoso de la muestra y $950 \mu \mathrm{L}$ de la mezcla buffer acetato: TPTZ: hierro férrico (10:1:1); se dejó reposar durante 10 minutos a temperatura ambiente. Se usó una solución de sulfato ferroso como referencia de $100-750 \mu \mathrm{M}$.

\section{RESULTADOS Y DISCUSIÓN}

\section{Características físicas}

Las cinco muestras de Coffee arabica obtenidas de cinco regiones que geográficamente se ubican en la zona nororiente (Cajamarca), en la zona selva central (Ucayali y Junín), en la zona central sur (Ayacucho) y suroriente (Cusco), tienen en común la temperatura media anual que va desde $23,6^{\circ} \mathrm{C}$ a $26,1^{\circ} \mathrm{C}$, con un promedio de $24,6^{\circ} \mathrm{C}$, aunque la altitud es variada y va desde 700 m.s.n.m. hasta 1760 m.s.n.m.

Los resultados de los sólidos solubles y la densidad aparente son homogéneos y no presentan diferencias significativas (tabla 1). Los valores obtenidos expresan que las cinco muestras contienen sustancias cuantitativamente muy cercanas. 
Tabla 1. Propiedades físicas de los extractos de Coffea arabica procedente de cinco regiones del Perú.

\begin{tabular}{lcc}
\hline & Sólidos solubles & Densidad aparente \\
& grados Brix $(\% \mathbf{p} / \mathbf{p})$ & $\mathbf{g} / \mathbf{m L}$ \\
\hline Ayacucho & 0,50 & 1,009 \\
Junín & 0,53 & 1,017 \\
Ucayali & 0,50 & 1,016 \\
Cajamarca & 0,53 & 1,017 \\
Cusco & 0,50 & 1,014 \\
\hline
\end{tabular}

Con estos datos, y considerando que todas fueron sometidas al mismo procedimiento de secado, tostación y molienda, las propiedades físicas de densidad aparente y sólidos solubles no mostraron diferencias significativas (tabla 1). Los procesos seguidos hasta la obtención de la molienda pueden modificar algunas propiedades físicas, entonces las modificaciones que pudieron haber por efecto de tratamiento no afecto a estas propiedades físicas ${ }^{12}$. En este sentido, la temperatura sería uno de los factores ambientales determinantes para la producción homogénea de compuestos bioactivos como los fenoles, flavonoides y probablemente otros metabolitos.

\section{Compuestos bioactivos no enzimáticos}

El contenido de fenoles totales (CFT) y flavonoides $(F)$ que se presentan en la figura 1 no muestra diferencias significativas, importante también es notar que este contenido similar se refleja en la relación F/CFT. Estos resultados son similares aun cuando proceden de regiones del norte, centro y sur del país, de altitudes que van desde los 700 m.s.n.m. hasta los 1760 m.s.n.m.

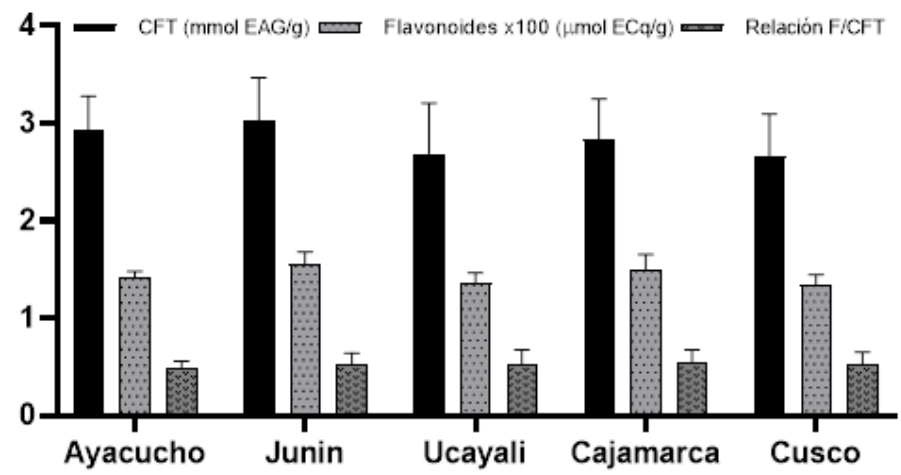

Figura 1. Contenido de fenoles totales (CFT) y flavonoides (F) de extractos de café tostado procedentes de cinco regiones. No hubo diferencias significativas. 
La ausencia de diferencias significativas en el contenido de fenoles totales (figura 1), refleja que la producción de estos compuestos orgánicos podría ser también consecuencia importante de la temperatura, la que debe ser entre $17^{\circ} \mathrm{C}$ y $26^{\circ} \mathrm{C}$. Otro estudio en café verde y procesado de las especies Coffea arabica y Coffea canephora tampoco hallaron diferencias significativas en el contenido de fenoles totales ${ }^{13}$.

El estudio de 34 muestras de cafés comerciales de Panamá, sobre el CFT de las muestras de cafés puros y mezclados estuvo en el rango de 0,17 a 0,28 y 0,07 a 0,10 mmol EAG/g, respectivamente ${ }^{14}$. Es decir, que el contenido de polifenoles totales del Coffea arabica procedente de los cinco valles de Perú es superior a los comerciales de Panamá, los estudiados estuvieron entre 2,7 y 3,0 mmol EAG/g de café. El factor tiempo de almacenamiento de los cafés comerciales, pudo haber modificado el contenido de fenoles, así se demostró la reducción significativa $(\mathrm{p}<0,01)$ en el estudio de almacenamiento durante doce meses a $5^{\circ} \mathrm{C}{ }^{15}$. (Król, Gantner, Tatarak, \& Hallmann, 2020). Otro resultado en Coffea arabica de Brasil, Colombia, Etiopía e India, mostraron un contenido de fenoles totales de $0,24,0,22,0,25$ y 0,25 $\mathrm{mmol} / \mathrm{g}$ de café, respectivamente ${ }^{16}$. También hay estudios del contenido de fenoles totales y flavonoides en bebidas de café preparadas de diversas formas. Se mostró que el preparado como "expreso" tuvo el mayor contenido de fenoles totales, $0,29 \mathrm{mmol} \mathrm{EAG/g} \mathrm{de} \mathrm{café} \mathrm{y} \mathrm{de}$ flavonoides $379 \mu \mathrm{mol} \mathrm{ECq} / \mathrm{g}$ de café y se asoció a una mayor capacidad antioxidante ${ }^{17}$.

Los flavonoides mostraron el mismo comportamiento que los fenoles, no hubo diferencias significativas, pero se notó un contenido ligeramente mayor (15\%) en la muestra de Junín $(155,0 \mu \mathrm{mol} \mathrm{ECq} / \mathrm{g})$ con respecto a Ucayali y Cusco $(135 \mu \mathrm{mol} \mathrm{ECq} / \mathrm{g})$. El mismo estudio de 34 muestras de café comerciales de Panamá ${ }^{14}$, mostró que el contenido de flavonoides fue de 76,3 a 132,0 y 32,2 a $50,0 \mu \mathrm{mol} \mathrm{ECq} / \mathrm{g}$, para los puros y mezclados, respectivamente. El análisis de correlación de Pearson, entre estos metabolitos secundarios, demostró una fuerte asociación $(\mathrm{r}=0,953)$ con un valor significativo $\mathrm{p}<0,05$. (tabla 2 ) lo que ratifica la homogeneidad de los contenidos.

\section{Capacidad Antioxidante Total}

La evaluación de la CAT frente a técnicas empleando radicales libres, $A B T S^{+}$y $D P P H$ no dio diferencia significativa entre Ayacucho, Junín, Ucayali y Cajamarca, solo en el caso de la CAT del Cusco tiene un menor valor con diferencia significativa $(p<0,05)$ frente a la muestra de Junín. Esta diferencia se muestra frente a ambos radicales libres y expresados con dos estándares, Trolox y ácido ascórbico (figura 2). La muestra procedente de Junín corresponde a una altitud de 700 m.s.n.m y la de Cusco corresponde a una altitud de 1047 m.s.n.m. 


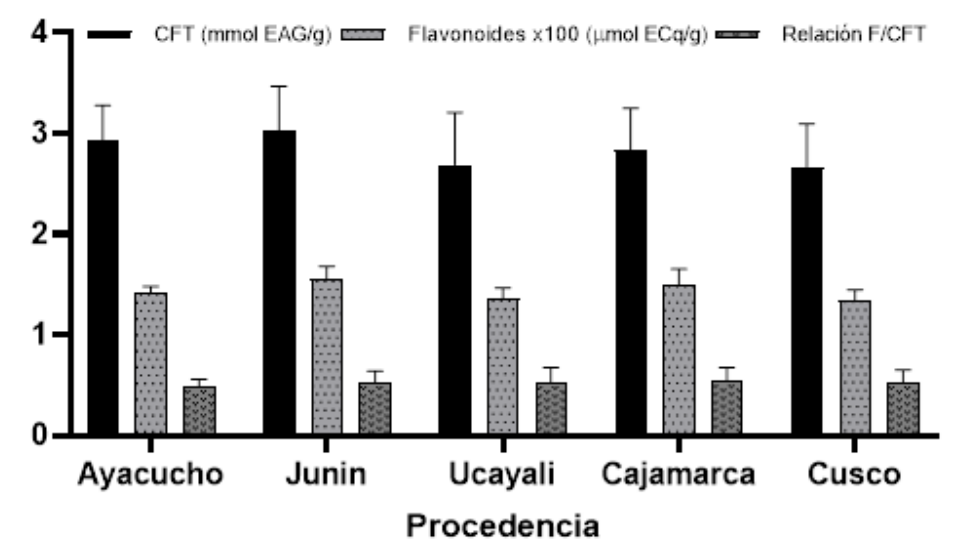

Figura 2. Capacidad antioxidante total (CAT) frente a radicales libres de extractos de café procedentes de cinco regiones del Perú. TEAC: capacidad antioxidante total equivalente a Trolox. AAEAC: capacidad antioxidante total equivalente a ácido ascórbico. *significancia $\mathrm{p}<0,05$.

Estos compuestos bioactivos no enzimáticos son directamente asociados al comportamiento antioxidante y por ende a los beneficios que de ellos derivan. Los extractos sometidos a las pruebas de CAT lo demostraron. En la figura 2 solo se observó diferencia significativa entre la muestra procedente de Junín (TEAC-ABTS: $62 \mathrm{mg} / \mathrm{g}$; TEAC-DPPH: $52 \mathrm{mg} / \mathrm{g}$ ) frente a la muestra de café procedente de Cusco (TEAC-ABTS: $55 \mathrm{mg} / \mathrm{g}$; TEAC-DPPH: $44 \mathrm{mg} / \mathrm{g}$ ). Estudios similares fueron hallados por Vega, De León y Reyes (2017), la TEAC-DPPH fue de 28 a $50 \mathrm{mg} / \mathrm{g}$ y de 6 a $15 \mathrm{mg} / \mathrm{g}$, para los cafés puros y las mezclas, respectivamente. Ya que temperaturas menores a $17^{\circ} \mathrm{C}$ o mayores a $26^{\circ} \mathrm{C}$ afectan la calidad de los granos.

Otro estudio en granos de Coffee arabica, como café verde y tostado de diferentes estados de México, mostraron valores de TEAC-ABTS de 47 a $63 \mathrm{mg} / \mathrm{g}^{18}$, los que se muestran en el presente estudio exhibieron valores de TEAC-ABTS más homogéneos en el rango de 55 a $62 \mathrm{mg} / \mathrm{g}$. Que pueden explicarse por las diferentes condiciones ambientales entre el Perú y México. 


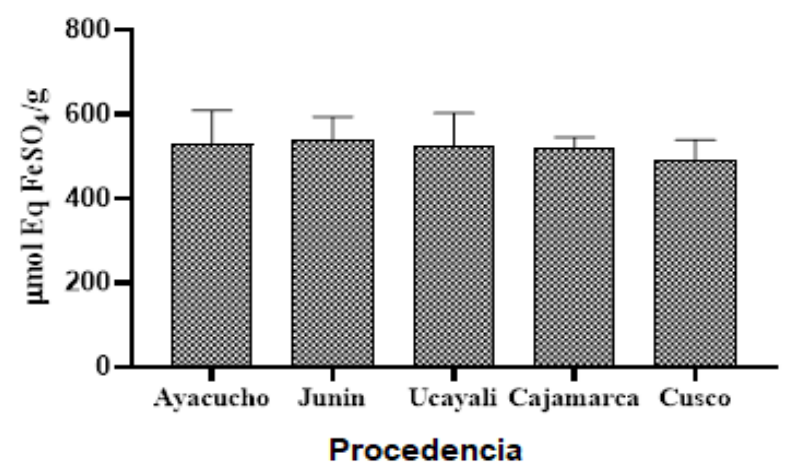

Figura 3. Potencial antioxidante reductor de ion férrico (FRAP) de extractos de café tostado procedente de cinco regiones del Perú. Sin diferencias significativas.

Sin embargo, cuando se ha evaluado el comportamiento por su capacidad antioxidante mediante la reducción del $\mathrm{Fe}^{+3}$ (FRAP) no se ha observado ninguna diferencia significativa (figura 3).

Tabla 2. Correlación de Pearson de los parámetros antioxidantes, fenoles y flavonoides de las muestras de café procedentes de las cinco regiones del Perú.

\begin{tabular}{lcccccc}
\hline & $\begin{array}{c}\text { AAEAC- } \\
\text { ABTS }\end{array}$ & \multirow{2}{*}{ FRAP } & $\begin{array}{c}\text { TEAC- } \\
\text { DPPH }\end{array}$ & $\begin{array}{c}\text { AAEAC- } \\
\text { DPPH }\end{array}$ & \multirow{2}{*}{ CFT } & F \\
\hline TEAC-ABTS & $\mathbf{0 , 9 5 1}$ & $\mathbf{0 , 7 6 6}$ & $\mathbf{0 , 9 5 5}$ & $\mathbf{0 , 9 5 5}$ & $\mathbf{0 , 9 5 1}$ & $\mathbf{0 , 9 5 9}$ \\
AAEAC-ABTS & & $\mathbf{0 , 8 7 5}$ & $\mathbf{0 , 9 9 9}$ & $\mathbf{0 , 9 9 9}$ & $\mathbf{1 , 0 0}$ & $\mathbf{0 , 9 9 9}$ \\
FRAP & & & 0,873 & $\mathbf{0 , 8 7 3}$ & $\mathbf{0 , 8 7 5}$ & $\mathbf{0 , 6 8 8}$ \\
TEAC-DPPH & & & & 1,00 & $\mathbf{0 , 9 9 9}$ & $\mathbf{0 , 9 5 5}$ \\
AAEAC-DPPH & & & & & 0,999 & $\mathbf{0 , 9 5 5}$ \\
CFT & & & & & & 0,953
\end{tabular}

CFT: contenido de fenoles totales; F: flavonoides; TEAC: capacidad antioxidante equivalente al Trolox, AAEAC: capacidad antioxidante equivalente al ácido ascórbico.

El análisis de correlación para la determinación de la asociación entre el contenido de compuestos bioactivos no enzimáticos y la capacidad antioxidante total mostraron una fuerte correlación $(>0,7)$ en toda la evaluación (tabla 2).

El CFT y flavonoides guardan una fuerte correlación con la actividad antioxidante evaluada con las tres técnicas (tabla 2), analizados con la correlación de Pearson ${ }^{19}$, especialmente entre CFT frente a las técnicas con radicales libres. Esta fuerte correlación expresa que los fenoles 
participan por mecanismos de donación de electrones (SET: single electron transfer) y por donación de átomo de hidrógeno (HAT: hydrogen atom transfer), así como también como agentes reductores ${ }^{20}$.

La importancia de los resultados de estas muestras procedentes de las cinco regiones del país radica en saber que, a pesar de las distancias geográficas y la altitud, las muestras de café de las cinco regiones contienen fenoles totales y flavonoides sin diferencia significativa, este contenido está fuertemente asociado a su CAT a través de los mismos mecanismos. Será necesario ahondar los estudios sobre estos mismos parámetros en el grano verde, pues es sabido que el proceso de tostación influye, además de las características organolépticas, en la composición y CAT ${ }^{21}$. Este trabajo constituye un punto de partida para la evaluación del comportamiento antioxidante in vitro de las diversas variedades de café cultivados en el territorio nacional, proyectando además estudios sobre la composición y otros ensayos in vivo.

\section{CONCLUSIONES}

El café peruano exhibe propiedades antioxidantes como las reportadas para esta especie en otros países, y se correlaciona con el contenido de polifenoles y flavonoides. La procedencia de las muestras de Coffee arabica de las cinco regiones del Perú, diferentes en ubicación y altitud, no ha determinado una diferencia significativa en el contenido de fenoles totales y flavonoides, ni en la capacidad antioxidante total.

\section{AGRADECIMIENTO}

Los autores agradecen el apoyo parcial de la Facultad de Medicina de la UNMSM en la ejecución del estudio; asimismo, a la Cámara Peruana del Café y el Cacao por las orientaciones recibidas para la obtención de las muestras. También se agradece a la empresa IMSA por las facilidades brindadas para la tostación y molienda. Finalmente, agradecemos al señor Rolando Gamarra, técnico del laboratorio del CIBN, por su trabajo más allá del horario establecido.

\section{REFERENCIAS BIBLIOGRÁFICAS}

1. Poole R, Kennedy OJ, Roderick P, Fallowfield JA, Hayes PC, Parkes J. Coffee consumption and health: umbrella review of meta-analyses of multiple health outcomes. BMJ. 2017 Nov 22;359: j5024. doi: 10.1136/bmj.j5024

2. Mojica BE, Fong LE, Biju D, Muharram A, Davis IM, Vela KO, Forester SC. The Impact of the Roast Levels of Coffee Extracts on their Potential Anticancer Activities. J Food Sci. 2018; 83(4): 1125-1130. 
3. Bean C, Nolte GE. Peru Coffee Annual. USDA Foreign Agricultural Services. Global Agricultural Information Network; 2016.

4. Haile M, Kang W. Antioxidant activity, total polyphenol, flavonoid and tannin contents of fermented green coffee beans with selected yeasts. Fermentation. 2019; 5(1):29. doi: 10.3390/fermentation5010029.

5. Canet G, Soto C, Ocampo P, Rivgera J, Navarro A, Guatemala G, Villanueva S. La situación y tendencias de la producción de café en América Latina y el Caribe. San José, Costa Rica: IICA; 2016.

6. Ramos E, Lima I, Cornejo G. Comparativo de calidad organoléptica de café (Coffea arabica L.) en Puno - Perú y La Paz - Bolivia. Rev Investig Altoandin. 2019; 21(2): 283-292.

7. Singleton V, Orthofer R, Lamuela-Raventos RM. Analysis of total phenols and other oxidation substrates and antioxidants by means of Folin-Ciocalteu Reagent. Methods Enzymol. 1999; 299: 152-178.

8. Zhishen J, Mengcheng T, Jianming W. The determination of flavonoid contents in mulberry and their scavenging effects on superoxide radicals. Food Chem. 1999; 64: 555-559.

9. Re R, Pellegrini N, Proteggente A, Pannala A, Yang MA, Rice-Evans C. Antioxidant Activity Applying an Improved ABTS Radical. Free Radic Biol Med. 1999; 26(98): 1231-1237.

10. Joyeux M, Mortier F, Fleurentin J. Screening of antiradical, antilipoperoxidant and hepatoprotective effects of nine plant extracts used in Caribbean folk medicine. Phytother Res. 1995; 9(3): 228-230.

11. Benzie I, Strain J. The ferric reducing ability of plasma (FRAP) as a measure of "Antioxidan power": the FRAP assay analytical biochemistry. Anal Biochem. 1996; 239: 70-76.

12. Nakilcioğlu-Taş E, Ötleş S. Physical characterization of arabica ground coffee with different roasting degrees. An Acad Bras Ciênc. 2019; 91(2). e20180191. doi: 10.1590/0001-3765201920180191

13. Pérez LM, Chávez K, Medina LA, Gámez N. Compuestos fenólicos, melanoidinas y actividad antioxidante de café verde y procesado de las especies Coffea arabica y Coffea canephora. BIOtecnia. 2013; 15(1): 51. doi:10.18633/bt.v15i1.136

14. Vega A, De León JA, Reyes SM. Determinación del Contenido de Polifenoles Totales, Flavonoides y Actividad Antioxidante de 34 Cafés Comerciales de Panamá. Inf Tecnol. 2017; 28(4): 29-38.

15. Król K, Gantner M, Tatarak A, Hallmann E. The content of polyphenols in coffee beans as roasting, origin and storage effect. Eur Food Res Technol. 2020; 246(1): 33-39.

16. Dybkowska E, Sadowska A, Rakowska R, Dębowska M, Świderski F, Świąder K. Assessing polyphenols content and antioxidant activity in coffee beans according to origin and the degree of roasting. Rocz Panstw Zakl Hig. 2017; 68(4): 347-353.

17. Ormaza-Zapata AM, Díaz-Arango FO, Rojano BA. The effect of pressure filtration coffee preparation methods (Coffea arabica 1. var. castillo) on antioxidant content and activity, and beverage acceptance. DYNA (Colombia). 2019; 86(209): 261-270. 
18. Lazcano E, Trejo MA, Vargas G, Pascual S. Contenido de Fenoles, Cafeína y Capacidad Antioxidante de granos de café verdes y tostados de diferentes Estados de México. Rev Iber Tecnología Postcosecha. 2015; 16(2): 293-298.

19. Ratner B. The correlation coefficient: Its values range between $+1 /-1$, or do they? J Target Meas Anal Mark. 2009; 17(2): 139-142.

20. Liang N, Kitts D. Antioxidant property of coffee components: Assessment of methods that define mechanism of action. Molecules. 2014;19(11):19180-19208.

21. Cho AR, Park KW, Kim KM, Kim SY, Han J. Influence of roasting conditions on the antioxidant characteristics of colombian coffee (Coffea arabica L.) beans. J Food Biochem. 2014; 38(3):271-280. 